- Modern techniques for preparing the root canal involve a crown-down approach to more efficiently remove infected debris and to improve access for irrigants.

- The balanced-force technique, with a $60^{\circ}$ clockwise turn followed by a balanced anti-clockwise cutting motion, is accepted as the most efficient method of file manipulation, (except that when using Hand GT files the motions are reversed).

- Copious irrigation with an appropriate antiseptic material is essential to clean the root canal system following shaping of the main canals.

- The smear layer should be removed with an EDTA solution before placing an intervisit dressing, or carrying out obturation.

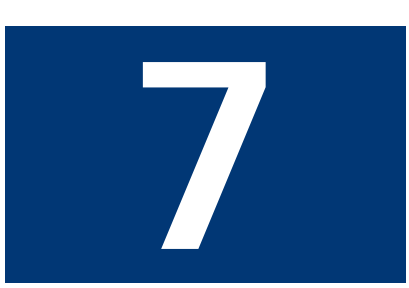

\title{
Endodontics: Part 7 Preparing the root canal
}

\section{P. Carrotte ${ }^{1}$}

Research into root canal preparation has led to significant changes in instrumentation techniques. Hand files should be manipulated by the balanced-force technique. Recent designs of endodontic instruments have variable tapers giving improved shaping ability. Nickel-titanium rotary instruments will rapidly and safely open the main root canals creating deep space to permit full permeation of irrigant solutions. Practitioners considering changing their endodontic technique are advised to attend hands-on practical courses to gain competence before using these in clinical practice.

\section{ENDODONTICS}

1. The modern concept of root canal treatment

2. Diagnosis and treatment planning

3. Treatment of endodontic emergencies

4. Morphology of the root canal system

5. Basic instruments and materials for root canal treatment

6. Rubber dam and access cavities

7. Preparing the root canal

8. Filling the root canal system

9. Calcium hydroxide, root resorption, endo-perio lesions

10. Endodontic treatment for children

11. Surgical endodontics

12. Endodontic problems

${ }^{1 *}$ Clinical Lecturer, Department of Adult Dental Care, Glasgow Dental Hospital and School, 378 Sauchiehall Street, Glasgow G2 3JZ

${ }^{*}$ Correspondence to: Peter Carrotte

Email:p.carrotte@dental.gla.ac.uk

\section{Refereed Paper}

doi:10.1038/sj.bdj.4811823

(๑) British Dental Journal 2004; 197:

603-613
Success in endodontic treatment depends almost completely on how well the root canal is shaped and cleaned. This part will cover the principles of root canal preparation, irrigation, root length determination, intracanal medication, and temporary fillings.

There have been more developments in recent years in this aspect of endodontic practice than any other. New instruments have been developed, employing different metals and different engineering philosophies. There has been a significant move away from the ISO standard 2\% taper instrumentation.

Two root canal preparation techniques using hand instruments will be described in detail, as these are the standard techniques currently taught in most dental schools, and are considered to be the most efficient and suitable for clinical dental practice. Details are also given of the use of engine-driven rotary instruments.

\section{PRINCIPLES AND RECENT DEVELOPMENTS OF ROOT CANAL PREPARATION}

The principles of root canal preparation are to remove all organic debris and microorganisms from the root canal system, and to shape the walls of the root canal to facilitate that cleaning and the subsequent obturation of the entire root canal space. However, a tooth root rarely contains a single simple root canal. Accessory canals, lateral canals, fins, anastomoses between canals, and an apical delta all contribute to the root canal system, as shown in Part 1. The majority of these anatomical features are not accessible to instrumentation. An irrigant solution must be used which can be flushed through this system, will destroy the microorganisms and preferably dissolve organic debris at the same time. Thus the current concept of root canal preparation is not cleaning and shaping, but shaping and cleaning. The main root canals should be rapidly and efficiently shaped with instruments to permit thorough and extended cleaning of the entire pulpal system with the irrigant solution.

Once shaped and cleaned, the root canal system is obturated to prevent further ingress of microorganisms, both apically and coronally, and to entomb any remaining microorganisms to prevent their proliferation. Currently, the root canal filling material of choice is gutta-percha, which requires a gradual, even, funnel-shaped preparation with the widest part coronally and the narrowest part at the apical constriction, normally approximately $1.0 \mathrm{~mm}$ short of the root apex (Fig. 1). Wide, relatively straight canals are simple to prepare, but fine, curved canals can present considerable difficulties. In the past, a number of techniques have been described, all of which have been designed to produce a tapered preparation.

\section{DEVELOPMENT OF PREPARATION TECHNIQUES}

In order to fully understand the current techniques for canal preparation, it would be beneficial to look briefly at previous methods, and the associated problems which led to further development. Interestingly, in 1933 a paper was published in the dental literature recommending the use of maggots to consume and remove the necrotic 


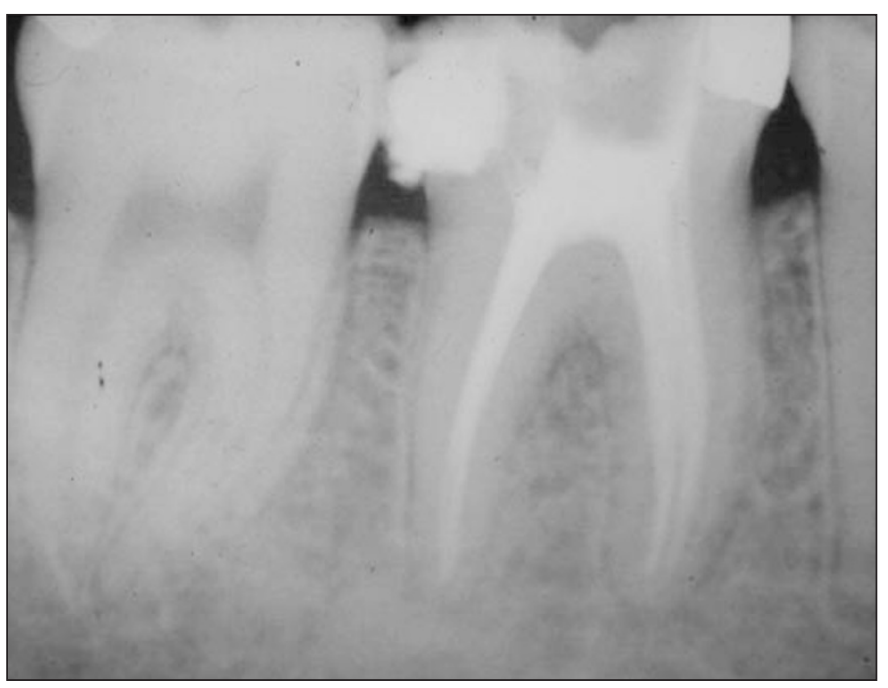

Fig. 1 The shape of the prepared root canal should be a gradual, even taper, with the widest part coronally and the narrowest part $1.0 \mathrm{~mm}$ from the root apex.

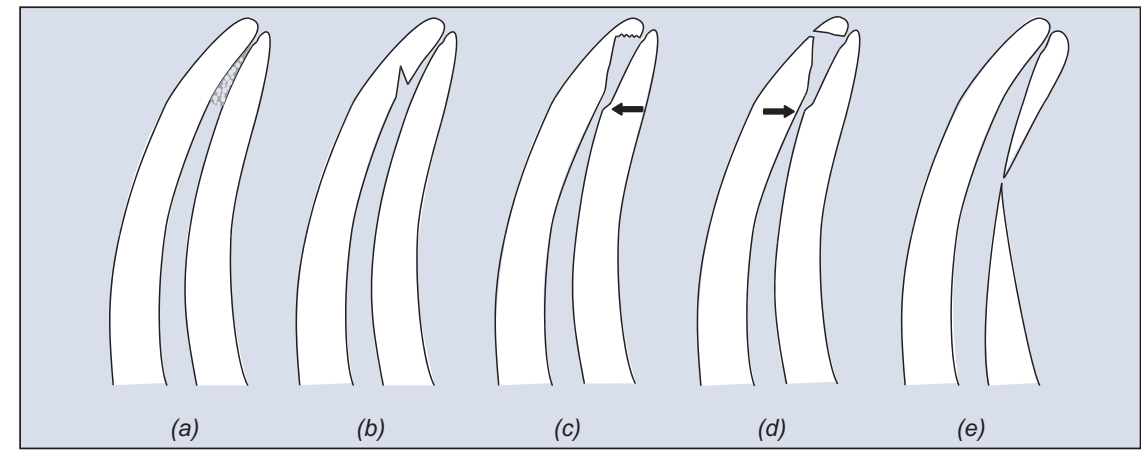

Fig. 2 Procedural errors in the preparation of curved canals. a) Dentine debris and pulp remnants packed into the apical part of the canal resulting in loss of working length. This may be avoided by recapitulation with fine files and copious irrigation. b) Ledging due either to not precurving the instrument, or forcing it into the canal. c) Apical zip caused by rotating the file excessively. d) Perforation due either to persistent filing with too large an instrument, or continual zipping. Note the narrower part of the canal in c) and d) is termed an elbow. This makes obturation of the root canal very difficult in the widened apical area. e) Strip perforation caused by overpreparing and straightening the curved canal. larger instrument is then inserted $1.0 \mathrm{~mm}$ less into the canal so that a taper is formed. In between placing each larger instrument, the master apical file is inserted to the working length to clear any debris collecting in the apical part of the canal; this is referred to as recapitulation.

The stepback technique helped to overcome the procedural errors of the standardised technique in slight to moderately curved canals, but in the more severely curved root canals problems still exist. There are three ways in which some of the problems of the curved root canal may be overcome, by using:

- A special filing technique.

- A file with a modified non-cutting tip.

- More flexible instruments.

\section{Stepdown technique}

This method, although not the term stepdown, was first suggested by Schilder in 1974, and the technique was described in detail by Goerig et al. ${ }^{2}$ It has been followed by other, similar techniques such as the double flared ${ }^{3}$ and the crown-down pressureless. ${ }^{4}$ The principle of these techniques is that the coronal aspect of the root canal is widened and cleaned before the apical part (Fig. 3). The obvious advantages of these methods over the stepback are as follows.

- It permits straighter access to the apical region of the root canal.

- It eliminates dentinal interferences found in the coronal two-thirds of the canal, allowing apical instrumentation to be accomplished quickly and efficiently.

- The bulk of the pulp tissue debris and microorganisms are removed before apical instrumentation is commenced, which greatly reduces the risk of extruding material through the apical foramen and causing periapical inflammation. This should reduce the incidence of after-pain following preparation of the root canal.

- The enlargement of the coronal portion first has several benefits. It allows better penetration of the irrigating solution to the entire root canal system and forms a reservoir of irrigant which is more readily replenished in the canal system. It also reduces the risk of compacting debris apically which may block the canal.

The stepdown technique is now the most widely used technique for canal preparation, and will be described later in this part. become less flexible and led to iatrogenic errors in curved root canals. Common problems encountered were ledging, zipping, elbow formation, perforation and loss of working length owing to compaction of dentine debris (Fig. 2).

\section{The stepback technique}

The stepback technique was devised to overcome the problem of the curved root canal and has been described by Mullaney. ${ }^{1}$ The apical region is first enlarged using files to a final master apical file size 25 or 30 ; each successively

\section{INSTRUMENT MANIPULATION}

In addition to the method of approach to the root canal, there have been numerous techniques for the manipulation of endodontic shaping instruments.

\section{Watchwinding and circumferential filing}

Watchwinding, or a continuous back and forth rotation with slight apical pressure, rapidly advances a fine file down a root canal. Each 


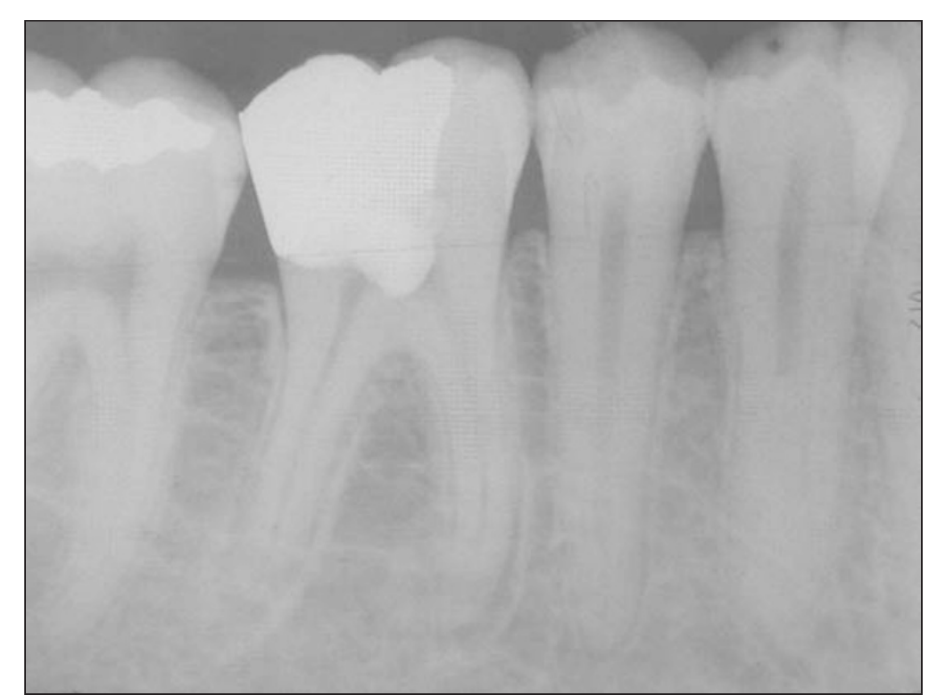

Fig. 3 The root canal in this tooth contains necrotic infected debris. The objective in endodontics is to remove the debris without extruding any through the apical foramen. It makes good sense to use a technique which cleans the coronal part first and then the apical portion.

slight turn engages the flutes of the file in the canal wall and removes dentine. Only fine files should be advanced to the apex in this way as there is a danger of compacting pulpal debris ahead of the file. If such a blockage occurs it can be extremely difficult to remove.

Once the file has reached the desired length, a push-pull filing action was used, moving the file circumferentially around the canal walls. When using K-type files an attempt was made to file on the outstroke only, again to reduce the apical compaction of debris. Hedstroem files were more efficient for circumferential filing, although these should not be used when watchwinding. Research into canal preparation found two distinct problems with circumferential filing.

The first was a tendency to preferentially file the inside wall of a curved canal. The technique of anticurvature filing was put forward by Abou-Rass et $a l .{ }^{5}$ Anticurvature filing involves filing predominantly away from the inner curve of a root to reduce the risk of a strip perforation. The mesiobuccal roots of maxillary molars and the mesial roots of mandibular molars are the teeth most frequently at risk. The method is used only in canals with a moderate-to-severe curve.

The second finding was that once the file engaged in the coronal part of the root canal, the apical flutes tended not to cut dentine but remain passive. The majority of the filing occurred coronally, which tended to leave underprepared canals which were not fully cleaned.

\section{The balanced force technique}

The balanced force technique, first described by Roane et al., ${ }^{6}$ is now the most widely taught technique for manipulating handfiles. It is particularly good when negotiating the curved root canal. The technique requires flexible files with non-cutting tips. The file is inserted into the canal until slight resistance is felt and then rotated $60^{\circ}$ clockwise to engage the flutes into the dentine. If a greater movement is made, iatrogenic problems can quickly arise. Using light apical finger pressure to hold the file at exactly the same depth in the canal, the file is now rotated through $360^{\circ}$ in an anticlockwise direction. The first $60^{\circ}$ of this turn cuts off the dentine engaged in the flutes of the file, and the remainder of the movement picks up this dentine in the flutes of the file prior to the next cycle. The amount of apical pressure required to rotate the file anticlockwise is just sufficient to prevent it from winding out of the canal. Watching the rubber stop in relation to the tooth assists in keeping the file steady with no in or out movement.

If the file is engaged too far into dentine with the first clockwise movement, ie if the turn is greater than $60^{\circ}$, excessive force is applied to the file during the cutting phase, and the file may fracture. The balanced force cycle of movement should be made no more than three times before the file is withdrawn to be cleaned, ideally by pressing it into a sterile sponge. The root canal system should be irrigated copiously before the file is reintroduced. Using this method, curved canals may be prepared to the full working length without producing apical transportation.

\section{Ultrasonic technique}

Ultrasound has been used to activate specially designed endodontic files. Ultrasound consists of acoustic waves which have a frequency higher than can be perceived by a human ear. The acoustic energy is transmitted to the root canal instrument, which oscillates at 20-40,000 cycles per second, depending on which unit is used. The superior cleaning effect is achieved by acoustic streaming of the irrigant and not, as originally thought, by cavitation. ${ }^{7}$ Irrigation with sodium hypochlorite is necessary, ${ }^{8}$ although some of the ultrasonic units are not designed to accept sodium hypochlorite through the system and, if water is used, they will be less efficient in their cleansing effect. Even when units designed to take sodium hypochlorite are used, daily maintenance must be carried out to prevent damage, particularly to metals, because the irrigant is corrosive.

The irrigant passes down the shank of the instrument and into the root canal, producing a continuous and most efficient system. Acoustic 


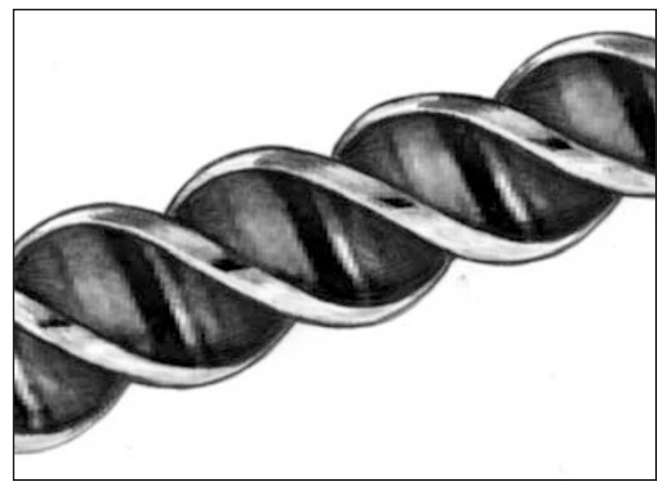

Fig. 4 A diagram of a nickel-titanium rotary file showing the concept of radial lands.

streaming is produced by the rapid file oscillations in the irrigant within the root canal. With more recent developments, ultrasonic machines are now largely used for their efficient irrigation properties rather than for canal shaping.

\section{Automated devices}

There have been many automated handpieces on the market over the years which claim to make the preparation of root canals quicker and more efficient. Although different designs and mechanical actions have been tried, they have all suffered from the inherent difficulties referred to earlier, caused by rotating or twisting conventional stainless steel instruments, such as zipping, perforation, canal transportation and broken instruments.

\section{Nickel-titanium}

However, the development of nickel-titanium alloys has revolutionised automated root canal preparation. The remarkable ability of these alloys to alter their crystalline state gives instruments manufactured from nickel-titanium profound flexibility. Mechanised instruments can withstand the distortions caused by repeated rotation in curved canals without causing preparation errors. Most of these instruments have design features such as radial lands (Fig. 4) to keep the instrument centred in the canal, and a non-cutting tip to guide the instrument down the canal. New designs are constantly appearing, (Fig. 5) and the clinician should ensure that considerable experience with whichever system is chosen has been obtained on extracted teeth, before the instruments are introduced into clinical patient treatment.

A controlled high-torque, low-speed motor is required for efficient use of the instruments. Most manufacturers of endodontic instruments produce such a motor, and their complexity may vary from that illustrated in Part 5, Figure 12, to that shown here in Figure 6. It must be emphasized that these nickel-titanium instruments do have a limited life, and will fracture in time after a large number of rotations. Slow (150-250 rpm) rotation does not impede their efficiency but extends their life. However, it is recommended that the instruments should be discarded after a certain number of cases as described by the

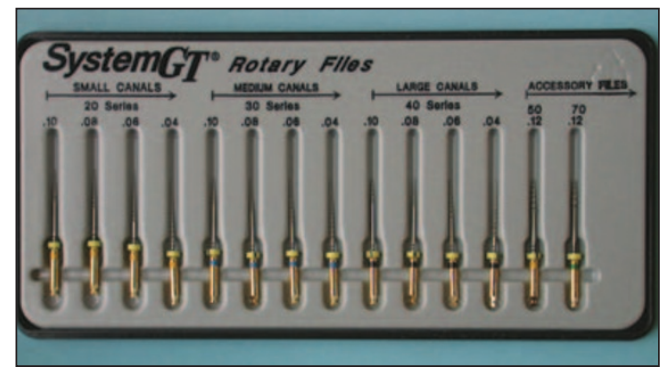

Fig. 5 One modern system of nickel-titanium rotary instruments - the System GT.

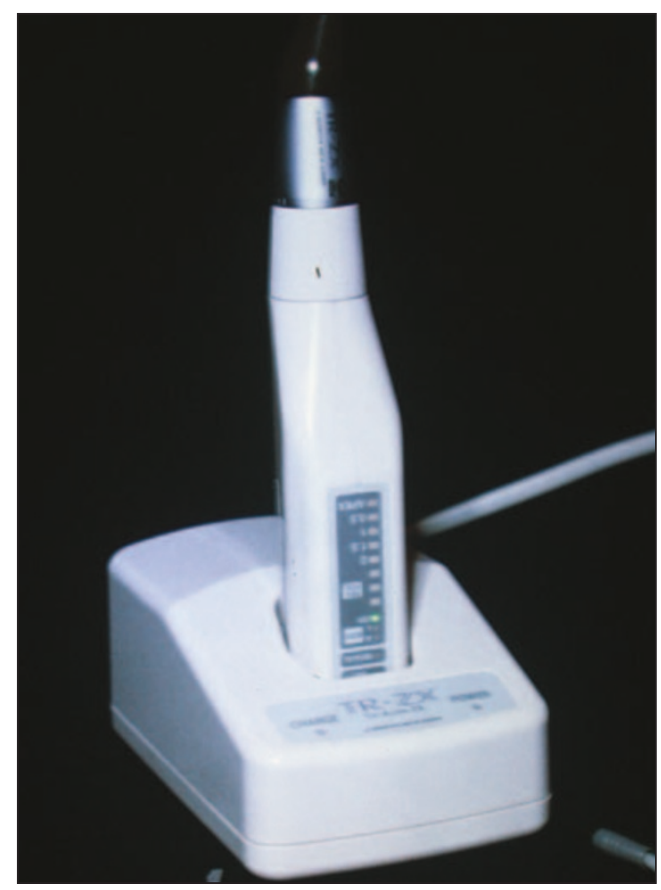

Fig. 6 A 'state-of-the-art' endodontic motor, being a rechargeable slow-speed, high-torque handpiece encompassing an apex locator and associated facilities.

manufacturer, and more frequently if an instrument has been used to negotiate difficult curved canals. The files should also be removed from the canal and cleaned frequently. Although debris is moved coronally it tends to compact in the file flutes, and if these become occluded the instrument will fracture. The separated part will engage in the root canal and may prove extremely difficult to remove.

\section{IRRIGATION}

\section{Sodium hypochlorite}

The importance of effective irrigation in root canal preparation cannot be overemphasised. A maxim in endodontics states that it is what you take out of a root canal that is important, not what you put in. Sodium hypochlorite is considered the most effective irrigant, as it is bactericidal, dissolves organic debris and is only a mild irritant. It must be clearly understood that almost any irrigant solution will cause an inflammatory reaction in the periapical tissues if it is expressed under pressure. ${ }^{9}$ Great care must be taken to follow the irrigation regime described below.

There is considerable debate about the recommended or optimum concentration of sodium 
hypochlorite. Ordinary domestic bleach, such as that purchased from any supermarket, has approximately 5\% available chlorine. This may be used neat, or may be diluted with purified water BP up to 5 times. Greater dilutions do not affect the antibacterial properties, but diminish the tissue dissolution property. Diluted solutions must therefore remain in the root canal for longer. Warming the irrigant makes it even more effective. ${ }^{10}$ There are other commercially available sodium hypochlorite products, but it must be emphasised that there should be no other additives, particularly sodium chloride.

During preparation, the root canal should be kept wet, with copious irrigation used after each instrument. The irrigant in the canal is only replaced to the depth of insertion of the needle. The needle must remain loose in the canal while the irrigant is being injected, to prevent the solution being expelled under pressure into the periapical tissues. To obtain total replacement of irrigant solution in the root canal, the smallest needle available (30-gauge) should be placed at the apical foramen. Obviously, this is a most hazardous procedure and it is suggested that the irrigation needle is only inserted to a maximum depth of $2.0 \mathrm{~mm}$ short of the working length. A file may then be worked in the apical $2.0 \mathrm{~mm}$, to stir and withdraw the dentine debris further into the canal, so that it can be flushed away. There are several differently designed irrigation needle tips, but in the author's opinion these are of little importance compared with the diameter of the needle. Whatever the tip design, unless the needle can penetrate loosely to the correct depth in the root canal, irrigation, however copious, will not remove dentinal debris.

\section{Chlorhexidine}

As referred to in Part 5, some practitioners have concerns about the use of sodium hypochlorite and prefer to use a solution of chlorhexidine. Whilst this has a similar antibacterial spectrum, it does not have the ability to dissolve organic debris and may not clean the entire root canal system as effectively. However, chlorhexidine does exhibit substantivity (adherence to dentine) and there is some evidence to suggest that it may be a more appropriate irrigant for retreatment of failed orthograde cases where sodium hypochlorite was the original irrigant.

\section{Ethylene-diamene tetracetic acid (EDTA) solution}

Endodontic instrumentation creates a smear layer on the root canal walls, particularly when using nickel-titanium rotary instruments. This smear layer occludes the dentinal tubules, and may protect microorganisms from the effects of the sodium hypochlorite irrigation. Flushing the canal with EDTA solution (Fig. 7) periodically during instrumentation removes the smear layer, and enables more effective cleansing. The final irrigation should always be with sodium hypochlorite.

\section{LUBRICATION}

Proprietory pastes containing EDTA are available, in combination with various agents, which greatly help instrumentation by chelating and softening the dentine. If a small portion is dispensed near to the file storage system, a little may be picked up on the tip of each new file as it is selected (Fig. 8).

\section{DETERMINATION OF ROOT CANAL LENGTH}

The exact apical termination of the root canal preparation has always been a subject of contention. The pedantic answer is that the root canal should be prepared to that point where the 'inside' of the tooth becomes the 'outside'. Some authors suggest this is the apical constriction, some the cemento-dentinal junction, some suggest that the apical foramen should be enlarged, some suggest that the preparation be taken to an arbitrary point $1 \mathrm{~mm}$ from the radiographic apex. As described in Part 4, the apical anatomy may be complex, and the term apical delta may be more appropriate. This author considers that the preparation should be taken as close as possible to the cemento-dentinal junction, and that wherever possible the foramen of the major canal should be kept patent. (This technique is described later.) The other minor canals forming the apical delta will only be cleaned by antibacterial irrigation flushing through the entire canal system.

An estimate of the root length is made from the pre-operative radiograph taken with a parallel technique. However, confirmation of the actual working length is not carried out until the coronal preparation of the canal has been completed, as this may straighten a curved canal which would change a measurement that had been taken too early. Most operators now confirm the actual working length when the crowndown preparation has progressed to within 3 or $4 \mathrm{~mm}$ of the estimated working length. There are three accepted methods for this determination.

\section{Working length radiograph}

A file is placed carefully in the canal until it is within approximately $2.0 \mathrm{~mm}$ of the overall length. Before insertion the file should be precurved to the shape of the canal and gently manoeuvred into position, if necessary using a watchwinding technique and slight apical pressure. For accurate reading of the radiograph a size 15 file is usually necessary. A silicone stop

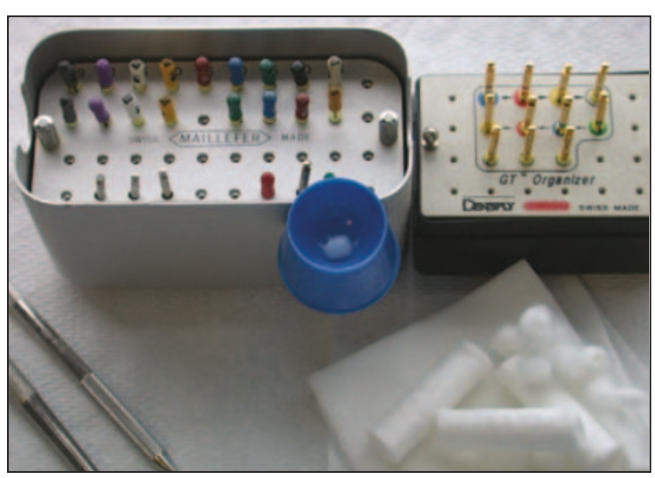

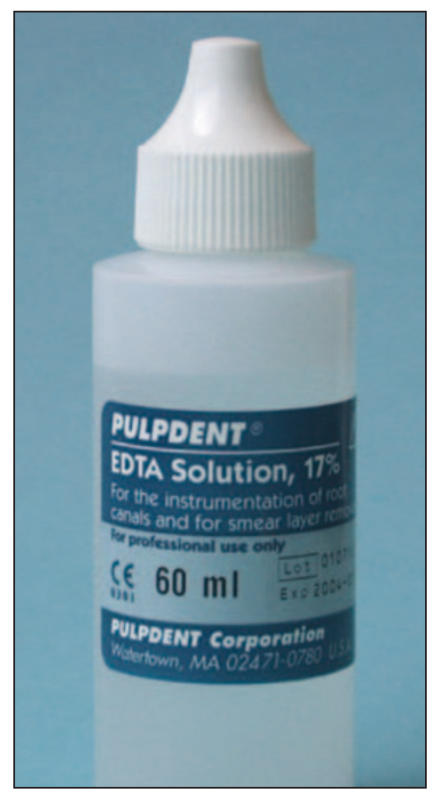

Fig. 7 EDTA solution for final flushing of the canal system.

Fig. 8 EDTA paste should be placed near the files and picked up on each instrument before use. 


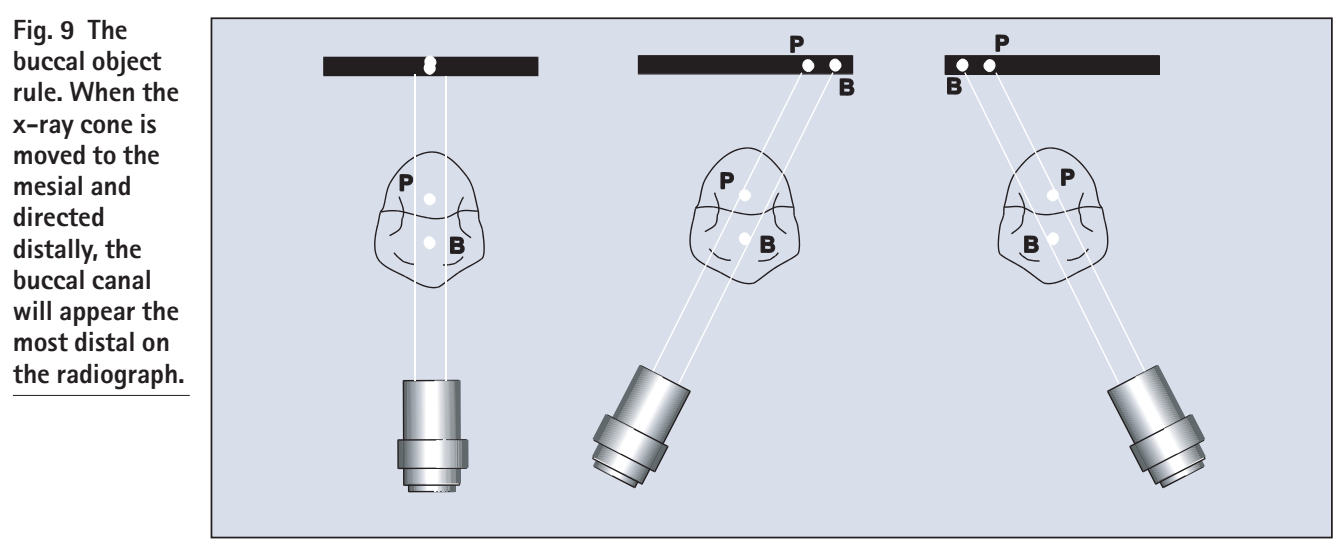

on the instrument shank is positioned against a reference point on the tooth, and both the length and the reference point should be noted in the records. When taking diagnostic radiographs, use should be made of the 'buccal object rule', where there are two or more canals present in the root (Figs 9 and 10). A second way of achieving the same result is to place a Hedstroem file in one canal and a K-file in the other, as the difference between the two is clear on the radiograph. The working length is calculated by measuring directly on the radiograph from the tip of the instrument to $1.0 \mathrm{~mm}$ short of the radiographic apex. It is only possible to estimate this arbitrary position using this technique.

\section{Electronic apex locators}

Electronic apex locators (seen in Part 5, Fig. 15) may be used as an alternative to a workinglength radiograph, assuming that a pre-operative film has been examined to obtain an estimated figure. These machines are capable of accurate measurement, and will give the location of the apical foramen. Apex locators are essential when a patient elects to have a minimum number of further radiographs taken. Many practitioners now use them routinely, particularly when the outline of the canal on the pre-operative film is indistinct or the canal curves towards or away from the radiograph beam. Modern apex locators work using different frequencies, determining the ratio between the different electric potentials proportional to each impedance. There is no longer any need to

Fig. 10 The diagnostic radiograph was taken from the mesial with the $x$-ray cone pointing distally. The buccal canal is therefore the distal one on the radiograph. dry the canal before use as they work in the presence of electrolytes. There is a distinct learning curve with their use, but it is usually apparent whether or not the measurement is in accordance with the original radiographic estimated working length. Errors may occur if there is a large coronal restoration or metallic crown causing a short circuit; if there is an open apex with a large periradicular lesion, or if there is a perforation. These are usually apparent and further measures should be taken.

In use, a file is inserted into the root canal and an electrical contact is made with the shank of the instrument. The device has a second electrode, which is placed in contact with the patient's oral mucosa. A digital display or audible signal shows when the tip of the instrument reaches the apical foramen. There is no doubt that modern apex locators can be even more accurate in length determination than a radiograph. ${ }^{11,12}$

\section{Tactile sensation}

An experienced clinician, armed with an accurate pre-operative parallel radiograph, can often feel the apical constriction with a fine instrument. If tactile sensation is in accord with the estimated length, further confirmation may not be necessary.

\section{PREPARATION OF THE ROOT CANAL}

Two techniques will be presented in detail, one using conventional hand instrumentation and one using nickel-titanium hand Files of Greater Taper. The stepdown technique has been modified slightly from the original description by Goerig. ${ }^{2}$ The use of nickel-titanium rotary instruments is presented in general terms only. As discussed earlier, there is a rapid development of these instruments, and the instructions and guidance of the manufacturers of the specific instruments chosen by the clinician should always be adhered to. It is strongly recommended that appropriate hands-on training is undertaken, practising the technique on a large number of extracted teeth before taking the new procedures to a patient.

\section{STEPDOWN TECHNIQUE WITH CONVENTIONAL 2\% TAPER INSTRUMENTS}

A pre-operative radiograph is taken, rubber dam placed and an access cavity prepared. The canal 
preparation is divided into two parts: i) coronal preparation, which permits radicular access for ii) apical instrumentation.

\section{Coronal preparation or radicular access}

First, the pulp chamber is copiously irrigated with sodium hypochlorite. Gates-Glidden burs are next introduced into the canal, directed apically and laterally away from the furcation. The larger sizes are introduced first, working sequentially further down the canal with smaller sizes. Some canals will accept a size 6 bur, but normally a size 4 would be used first, followed by a size 3 . Each bur will penetrate 2-3 $\mathrm{mm}$ further than the previous one. EDTA paste should be used with each bur, and the canal should be irrigated between each entry. Eventually, in a relatively straight canal, the No. 2 bur is inserted $10-12 \mathrm{~mm}$ into the canal from the occlusal reference point. In a curved canal the pre-operative radiograph should be checked for the maximum straight line penetration of the bur.

Gates-Glidden burs should be rotated with constant medium drill speed from the time they enter the canal until removed. Gates-Glidden burs must not be taken into a curve, or they will almost certainly fracture. If the shank of a bur does break, it usually does so near the handpiece head and may be retrieved easily from the tooth, as seen in Figure 11. However, if the head does become separated from the shank within the canal, removal may be extremely difficult. ${ }^{13}$

The bur may be flexed against the canal wall slightly on withdrawal to ensure that the natural shape of the canal is maintained. Thus a round canal will remain round, but an oval canal will be prepared to a smooth oval funnel. A wide oval or 'figure-of-eight' shape may need preparing at both extremities to produce a wide flare. Instrumentation with the stepdown technique in the radicular access is accomplished using only light pressure directed apically and away from the furcation, or perforation may result.

An alternative to Gates-Glidden burs is the use of standard flexible K-type files with safe tips, used with the balanced force technique. Following initial widening of the mouth of the canal only with a Gates-Glidden bur, the largest hand file which will enter the canal is selected and worked apically, using EDTA paste as a lubricant. Once penetration proves difficult the file should not be forced further, or fracture may result. The next size smaller file is selected, and sequentially smaller files used until the coronal preparation is complete.

\section{Apical preparation}

The coronal flaring already carried out makes access to the apical portion of the root easier, as there are no dentinal obstructions and access is more direct. Thus, once the coronal preparation is complete, flexible $\mathrm{K}$-type files with safe tips may be used sequentially with the balanced force technique previously described. A size appropriate to the particular canal and the final size Gates-Glidden drill is selected, perhaps a

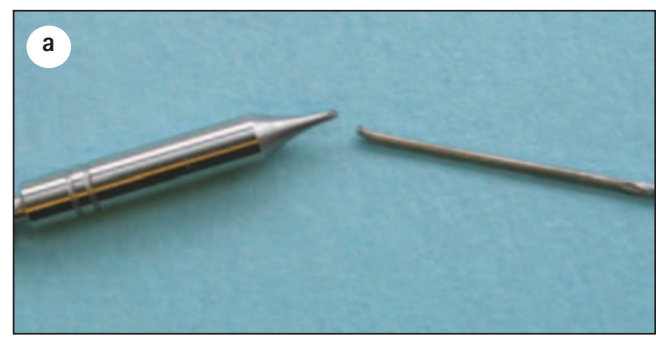

Fig. 11 A Gates-Glidden bur which fractured during use in the root canal, showing the normal point of separation, permitting removal with Steiglitz forceps. However, b) shows that this is not always the case.

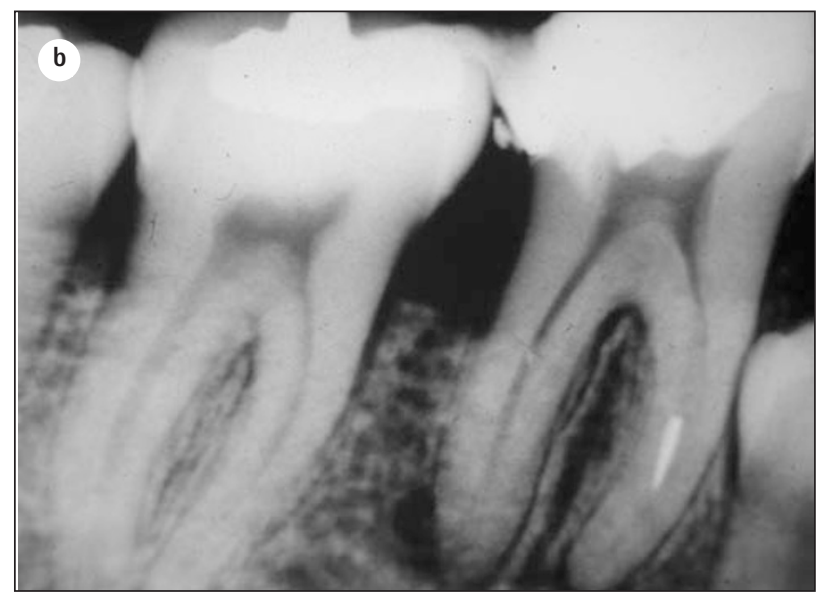

size 60, the tip dipped into a canal lubricant, and the instrument worked slightly further into the canal. Sequentially smaller files are selected until the canal is prepared to 3-4 $\mathrm{mm}$ short of the estimated working length. Now the actual working length must be confirmed by one of the methods described earlier, radiograph or apex locator. Once the working length has been confirmed, the apical preparation can be completed. In narrow or sclerosed canals the operator may prefer to take a small 08, 10 and 15 files to working length before commencing any canal preparation. The prepared canal then acts as guidance for the larger sizes. This procedure is illustrated diagrammatically in Figure 12.

\section{Stepback technique}

Following the preparation of the coronal part of the root canal, the apical preparation may also be carried out using the stepback technique. Starting with the size 15 file at the working

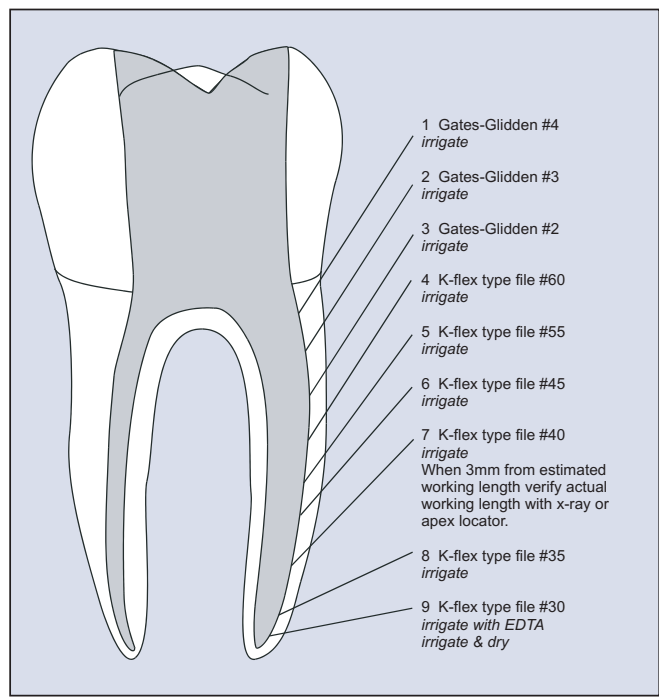

Fig. 12 A diagrammatic illustration of the sequence of instruments in a conventional $2 \%$ taper hand file canal preparation. 
Fig. 13 An illustration of the steps involved in the preparation of a simulated root canal with Hand Files of Greater Taper.

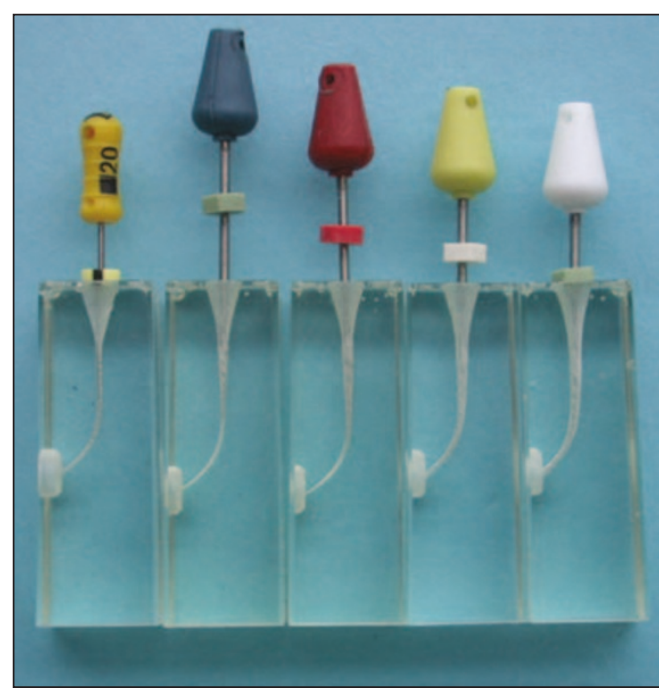

length, and progressing to sizes 20 and 25, an apical stop is made. Copious irrigation and recapitulation with fine files will prevent build-up of canal debris. The master apical file will usually be no greater than 25 or 30 . The apical portion of the canal is now tapered by stepping back. A file one size larger than the master apical file is worked with balanced force to $1.0 \mathrm{~mm}$ short of the working length. Each successively larger file

\section{Table 1 Root canal preparation using GT hand files}

\section{Aceess and coronal preparation}

- Access - Remove roof of pulp chamber and locate canal orifices with the use of safe ended access Bur and DG16. Ensure straight line access to the canal orifice.

- Irrigate pulp chamber with sodium hypochlorite

- Estimate working length from pre-operative radiograph.

- Using a gentle watchwinding technique, and EDTA chelating agent/lubricant (File Eze/Glyde), advance a \#08, \# 10, \#15 and \#20 K-type file to about $3 \mathrm{~mm}$ short of the estimated working length

- If desired, with irrigation with $\mathrm{NaOCl}$ and with the use of the lubricant, open up the canal orifice using Orifice Shaper 4 \& 3 crown down for approximately 3 or $4 \mathrm{~mm}$. Orifice Shapers should be rotated at $250 \mathrm{rpm}$ and used with a light pecking motion, keeping the instrument rotating within the canal at all times (Gates-Glidden drills can also be used for this procedure).

Mid-canal preparation

- Continue irrigation with $\mathrm{NaOCl}$ and the use of lubricant. Insert the \#12 (BLUE) GT hand file within the canal and using a reverse balanced force action $\left(60^{\circ}\right.$ turn anticlockwise followed by clockwise $180^{\circ}$ turn with apical pressure - the 'balanced force') progress the file until resistance is felt and no further advancement of the file is possible. Do not use excessive force, and remove and clean the file after every three cycles.

- In the same manner insert the \# 10 (RED) GT hand file, repeating the procedure to penetrate further down the cana

- Either repeat the process with the \#08 (YELLOW) hand file, or if approximately $3 \mathrm{~mm}$ from the estimated working length, proceed to:

Apical preparation

- Establish working length using electronic apex locator or with a diagnostic radiograph.

- Negotiate canal to full working length advancing \#15 and \#20 K-type files with watchwinding or balanced force motion.

Apical shaping

- Check canal patency using \# $15 \mathrm{~K}$-type file with copious irrigation of $\mathrm{NaOCl}$ and use the \#08 (YELLOW) GT hand file looking for resistance.

- Check canal patency before introducing the \#06 (WHITE) GT hand file, this file should progress to full working length.

- Apical gauging can be checked or further enlarged with the use of K-type files.

- Continue irrigation with $\mathrm{NaOCl}$ for a total preparation time of 30 minutes, followed by final rinse of EDTA solution.

The above procedural protocol may have to be adapted taking into account the apical constriction, working length, apical curvature and general canal anatomy. size is inserted $1.0 \mathrm{~mm}$ less than the previous size until the radicular access preparation is reached. In between each larger file selection the master apical file is inserted to full working length (recapitulation) and irrigation is used to remove all the debris.

\section{STEPDOWN TECHNIQUE WITH HAND FILES OF GREATER TAPER}

The stepdown technique may be modified with the use of this range of files described by Buchanan in $1996 .{ }^{14}$ The balanced force technique is used as described previously, except that these instruments are used in the opposite rotation to conventional files. It was considered that the crucial part of the balanced force technique is the cutting cycle. Right-handed clinicians (representing some $90 \%$ of the work-force) can make this movement more easily in a clockwise direction than anticlockwise. Thus the initial movement to engage the dentine with these files only is a $60^{\circ}$ turn anticlockwise, and the balanced force cutting motion is $360^{\circ}$ clockwise.

Following access to and irrigation of the pulp chamber the canal must first be gently explored to length with conventional 2\% taper hand files. A gentle watchwinding technique is used with size 08,10 and 15 files. The tip of the Greater Taper File then acts as a pathfinder rather than as a preparation file.

Using EDTA lubrication paste, the largest file with a blue handle and a taper of $12 \%$, is used first to gain coronal access. When resistance is met the instrument is not forced further apically, but the red handled 10\% taper file is used to penetrate further. The yellow handled $8 \%$ taper and white handled 6\% taper follow in sequence, until the canal is prepared to working length.

The technique is varied according to the clinical situation. In a wide, straight canal, only a single $12 \%$ taper file may be required. In a narrow, curved canal, the clinician may only use the smaller files, alternating them repeatedly to create space for further apical penetration. Before attempting to instrument narrow, curved canals it is again always advisable to use conventional $2 \%$ taper instruments from size 08 through to size 20. The tip of the Greater Taper file then acts as a pathfinder, dentine removal occurring at the side of the instrument, not the tip.

Once a smooth-tapered canal has been prepared, the clinician may consider it necessary in some cases to enlarge the apical constriction slightly with conventional hand files.

The technique is summarised in Table 1 , and shown in Figure 13.

\section{NICKEL-TITANIUM ROTARY TECHNIQUE}

The development and design of these instruments has been referred to earlier, and should be referred to in conjunction with this description of technique. Each manufacturer of these instruments produces a protocol for use for their own specific product. As it would not be appropriate to describe any single manufacturer's technique 
here, the description which follows must therefore be generic. The basic concepts are the same whatever the instrument chosen. The technique for use is crown-down, with copious irrigation. Indeed, these instruments conform totally to the stated objectives in modern root canal therapy, shaping the canal rapidly and efficiently so that thorough cleaning of the root canal system can be carried out with appropriate irrigants. The files must be used in a slow-speed, controlled torque motor, or they are prone to fracture.

\section{Coronal preparation or radicular access}

Nickel-titanium instruments cannot easily be precurved, and require straight line access to the root canal orifice. The use of ultrasonic tips to refine the access cavity has already been described. Nickel-titanium 'orifice shapers' have replaced Gates-Glidden drills, and may be used sequentially from the largest to the smallest sizes. These remain centred in the canal and will flare the canal walls to approximately halfway down the canal. Their use may be restricted in narrow or curved canals. They are used with a very light apical pressure, often described as the 'pencil-lead' pressure, ie that which would break the lead when using a propelling pencil. Each instrument should be used for no more than 5-10 seconds at a time before removing from the canal, cleaning, irrigating and adding lubricant.

\section{Apical preparation}

Once the coronal preparation is completed, the canal should be explored to full working length using fine hand files and the balanced force technique. The working length should be confirmed, and the canal enlarged to a size 15 or 20. If this is not done, the rotary instrument will have to cut at its tip, rather than along its length, which may lead to jamming and fracture. Each time a file is removed from a canal after use the position of the dentine debris in the flutes should be inspected. The instruments should cut along their entire length. If debris is only seen at the tip, the instrument may be excessively stressed, which may lead to fracture. The apical part of the canal may now be prepared with sequentially smaller instruments, stepping down the canal with each smaller size. It may be necessary to return to a larger size during preparation (recapitulation) to create more space for the smaller instruments.

\section{Summary of technique}

- Motor set at slow speed recommended for instrument, usually 150-250 rpm.

- Use each instrument for only 5-10 seconds.

- Light apical pressure, using either a gentle 'planing' pressure, or a slight 'pecking' motion depending upon the instrument design.

- Use EDTA lubricant with each instrument.

- Copious irrigation with sodium hypochlorite between instruments.

- Step down in sequence from the largest to the smallest. (NB This will depend upon which system is being used. If variable taper files have been selected, as in Fig. 5, then a 10\% taper file will be used until resistance is felt, moving to an $8 \%, 6 \%$ and $4 \%$ until any of these reaches working length, depending upon the canal size. Alternatively, if a single taper, variable tip system has been selected, the largest tip size will be used first, reducing sizes as the canal is negotiated until, once again, one instrument reaches working length.)

\section{FURTHER READING}

It must be stressed that the techniques described are generic, and that hands-on practice is essential, following the specific manufacturer's protocol until competence is achieved. The various instruments and techniques are described, compared and contrasted, in numerous publications, for example. ${ }^{15-18}$ The prudent clinician would be advised to refer to the endodontic literature before embarking on new purchases and clinical practice. A useful series of clinical articles was presented by Buchanan. ${ }^{19}$

\section{PATENCY FILING}

Research has shown that most canal preparation techniques lead to the extrusion of debris through the apical foramen. This is removed by the normal body defence systems, although a certain amount of inflammation will result. Concern has been expressed by some authorities that such debris may remain in the apical constriction, and may contribute to failure, particularly if it harbours microorganisms. ${ }^{20}$ The technique of patency filing involves passively inserting a small file, size 08 or 10, $2 \mathrm{~mm}$ beyond the established working length. No attempt is made to instrument the foramen, merely to keep it open or patent by deliberately extruding the debris into the periradicular tissues.

The literature on patency filing is at present quite equivocal. No research workers have been able to show either a decrease or an increase in post-operative symptoms or case prognosis. The technique remains subjective and subject to the operator's personal philosophy.

\section{INTRACANAL MEDICATION}

\section{Calcium hydroxide}

There is almost universal agreement that when an intervisit dressing is required, calcium hydroxide is the material of choice, and this is discussed in Part 9. There is far less agreement as to whether such dressings are indicated. Singlevisit endodontics - the shaping, cleaning and obturation of the root canals in one appointment - remains controversial. Most endodontists would agree that when the tooth under treatment is not infected, for example when performing elective endodontics or treating large exposures of vital pulps, completing treatment in a single visit is advisable. However, Sjögren et al. showed a significant increase in prognosis when infected root canals were dressed with calcium hydroxide for one week before obturation. ${ }^{21}$ Gutmann has suggested that this effect was only apparent because their research employed 1\% 
Fig. 14 A fine tipped plastic canula may be used to deliver medicaments deep into the root canal. Its size may be compared to the standard 28-gauge irrigating needle.

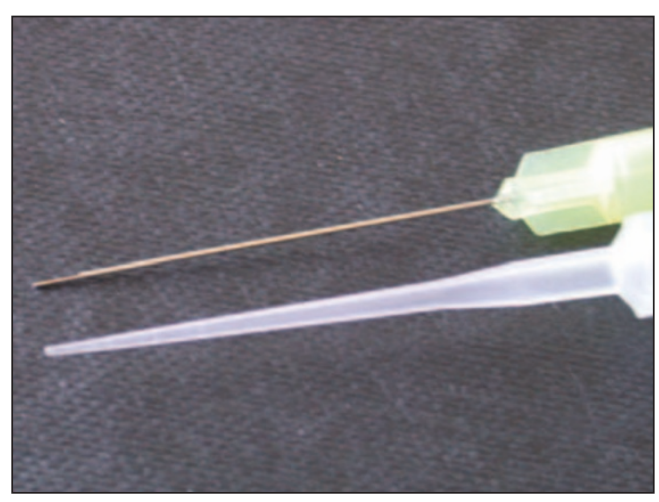

sodium hypochlorite, whereas the use of a full strength solution would preclude the need for such a dressing. ${ }^{22}$ The wise practitioner would consider which approach best suits his or her style of practice.

Calcium hydroxide is applied with a spiral paste filler (noting the caution given at Part 5, Fig. 20), or a fine-tipped syringe may be used as seen in Figure 14. Care should always be taken not to extrude the material beyond the apical constriction. If this happens, inflammation may result which could take several days to subside. Calcium hydroxide containing points are available from which it is postulated that ions will dissociate into the fluid in the root canal, and which may be better controlled in apical length. However, research supporting this has not yet been reported in the endodontic literature.

A recent addition to the range of intracanal dressing available is the mixture Vitapex, shown at Figure 15. As well as calcium hydroxide, the material contains iodoform. It may be indicated for deep-seated infections of the root canal, such as the upper canine, shown in Part 2, Figure 1, which had resulted in an extra-oral sinus beneath the patient's eye. This closed and the tooth healed following one week's dressing. Extreme care is needed to ensure that this viscous dressing has been fully removed from the canal walls before obturation.

\section{Steroids}

Steroids are readily obtainable in the form of triamcinalone (Ledermix paste). The use of the paste for dressing an inflamed vital pulp prior to commencing root canal therapy is discussed in Part 3. Some authorities recommend the use of the paste as an intervisit medicament paste when severe periradicular inflammation is present. It is suggested that it may be wiped on the canal wall using a file or paper point which is then withdrawn; a small amount is then placed on a pledget of cotton wool and sealed into the pulp chamber between appointments. The author prefers to mix the paste with a calcium hydroxide preparation, adding a little sterile water or local anaesthetic to make the mixture slightly more fluid. The canals may then be completely filled with this mixture prior to placing a temporary coronal seal.

Of course, no intracanal medicament yet exists that will sterilise the root canal, and the importance of biomechanical preparation cannot be stressed too strongly.

\section{TEMPORARY RESTORATIVE MATERIALS}

If endodontic treatment cannot be completed in one visit, it is essential that a temporary restorative material is used as an inter-appointment dressing that will not permit access to bacteria or oral fluids. Even when the root canal treatment has been completed, a well-sealed temporary restoration is necessary prior to the placement of a permanent restoration. The material should prevent contamination of the root canal system and must be sufficiently strong to withstand the forces of mastication.

Two different temporary materials are recommended. Of the many proprietary materials, Cavit has been shown to provide the best seal. ${ }^{23}$ Cavit provides a good seal, is simple to apply and quick to set. On the other hand, it lacks strength and will not stand up to masticatory forces. It should be confined to single surface fillings for periods not exceeding a week. However, the shape of an endodontic access cavity is not retentive, and all such materials will either leak or be lost entirely in time. Any ingress of microorganisms may reinfect a prepared canal, and the time saved by using a rapid technique may be severely lost if such leakage occurs. Thus the use of a glass-ionomer cement should be considered. It is adhesive, antibacterial, stands up well to forces of mastication and is more stable than other materials.

It is a useful routine, with an inter-appointment dressing, to place a sterile pledget of cotton wool in the pulp chamber, followed by a layer of gutta-percha. The temporary restorative material is then placed over the guttapercha (Fig. 16). At the next visit, a high-speed bur may be used to remove the temporary restoration without any danger of filling material lodging in the canal entrances or blocking the canals. The gutta-percha provides a base for the restorative material and prevents the bur becoming caught in the cotton wool when the temporary filling is removed.

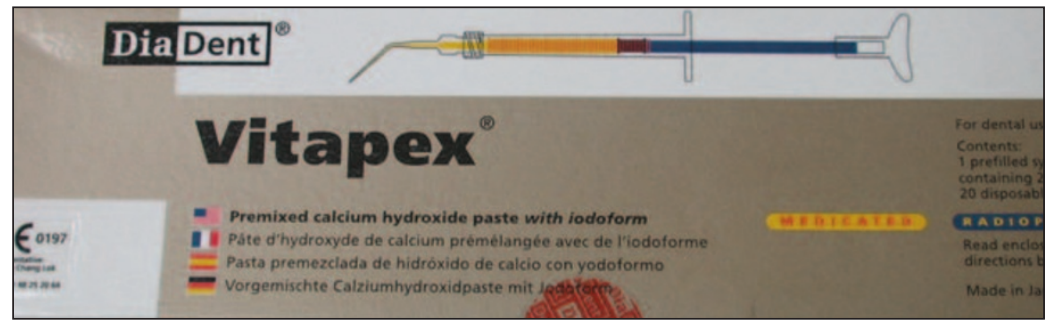




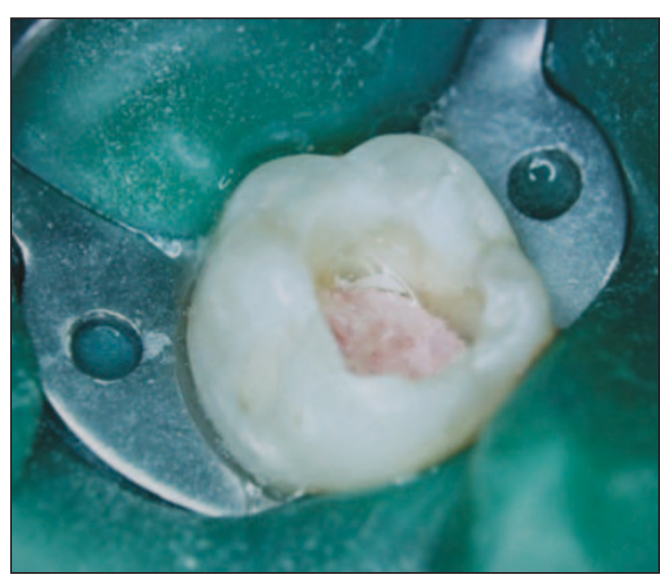

1. Mullaney T P. Instrumentation of finely curved canals. Dent Clin North Am 1979; 23: 575-592.

2. Goerig A C, Michelich R J, Schult H H. Instrumentation of root canals in molars using the stepdown technique. J Endod 1982; 8: 550-554.

3. Fava $L$ R. The double flared technique: an alternative for biomechanical preparation. J Endod 1983; 9: 76-80.

4. Morgan L F, Montgomery S. An evaluation of the crown-down pressureless technique. J Endod 1984; 10: 491-498.

5. Abou-Rass M, Frank A, Glick D. The anticurvature filing method to prepare the curved root canal. $J$ Am Dent Assoc 1980; 101: 792-794.

6. Roane J B, Sabala C L, Duncanson M G. The 'balanced force' concept for instrumentation of curved canals. J Endod 1985; 11: 203-211.

7. Ahmad M, Pitt Ford T, Crum L. Ultrasonic debridement of root canals: an insight into the mechanisms involved. J Endod 1987; 13: 93-101.

8. Griffiths B, Stock C. The efficiency of irrigants in removing root canal debris when used with an ultrasonic preparation technique. Int Endod J 1986; 19: $277-284$

9. Sabala C L, Powell S E. Sodium hypochlorite injection into periapical tissues. J Endod 1989; 15: 490-492

10. Berutti E, Marini R, A scanning electron microscope evaluation of the debridement capability of sodium hypochlorite at different temperatures. J Endod 1996; 22: 467-470.

11. Pagavino G, Pace R, Baccetti T. A SEM study of in vivo accuracy of the Root ZX electronic apex locator. J Endod 1998; 24: 438-441.

12. McDonald N J. The electronic determination of working length. Dent Clin North Am 1992; 36: 293-307.

13. Lumley P J. Management of silver points and fractured

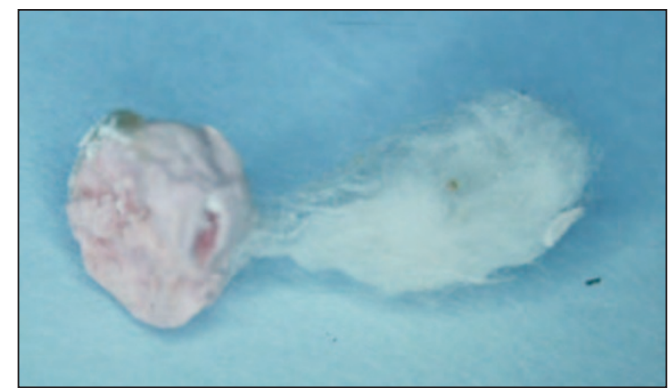

Fig. 16 A layer of gutta-percha temporary filling material has been placed to protect the root canals before the restorative material is applied. The inset shows how this may be removed and prevents fragments of the temporary filling entering the root canal.

instruments. CPD Dentistry 2000; 1: 87-92.

14. Buchanan L S. The art of endodontics: Files of greater taper. Dentistry Today 1996; 42: 44-49.

15. Kavanagh D, Lumley P J. An in vitro evaluation of canal preparation using Profile .04 and .06 taper instruments. Endod Dent Traumatol 1998; 14: 16-20.

16. Hulsmann M, Schade M, Schafers F. A comparative study of root canal preparation with HERO 642 and Quantec SC rotary Ni-Ti instruments. Int Endod J 2001; 34: 538-546.

17. Barbakow F, Lutz F. The Lightspeed preparation technique evaluated by Swiss clinicians after attending continuing education courses. Int Endod J 1997; 30: 46-50.

18. Bryant S T, Thompson S A, Al-Omari M A, Dummer P M H. Shaping ability of Profile rotary nickel-titanium instruments with ISO sized tips in simulated root canals. Int Endod J 1998; 31: (part 1) 275-281, (part 2) 282-289.

19. Buchanan L S. The standardised taper root canal preparation. Parts 1-6. Int Endod J. Part 1 2000; 33: 516-529; Part 2 2001; 34: 63-71; Part 3 2001; 34: 149-156; Part 4 2001; 34: 157-164; Part 5 2001; 34: 244-249; Part 6 2001; 34: 250-259;

20. Ruddle C J. in Cohen S and Burns R C, Pathways of the Pulp Eighth Edition, Page 276-277. St Louis: Mosby, 2002.

21. Sjögren U, Figdor D, Persson S, Sundqvist G. Influence of infection at the time of root filling on the outcome or endodontic treatment of teeth with apical periodontitis. Int Endod J 1997; 30: 297-306.

22. Guttman J L. Presentation to The British Endodontic Society Spring Scientific Meeting, October 2000, Chester, UK.

23. Anderson R W, Powell B J, Pashley D H. Microleakage of three temporary endodontic restorations. J Endod 1988; 14: 497-501. 